\title{
Optical fiber-driven low-energy electron gun for time-resolved streak diffraction
}

\author{
Chiwon Lee ${ }^{1}$, Günther H. Kassier ${ }^{1}$, and R. J. Dwayne Miller ${ }^{1,2, *}$ \\ ${ }^{1}$ Max Planck Institute for the Structure and Dynamics of Matters, Luruper Chausee 149, 22607 , \\ Hamburg, Germany \\ ${ }^{2}$ Departments of Chemistry and Physics, University of Toronto, M5S 3H6, Ontario, Canada
}

\begin{abstract}
The wave guiding feature of the optical fibre optical fibres is specifically exploited to construct a novel type of electron gun to realize single-shot low-energy electron diffraction experiments with the subpicosecond resolution for studying irreversible samples.
\end{abstract}

\section{Introduction}

In contrast to the conventional stroboscopic pump-probe scheme using hundreds of short bunches, the ultrafast streak diffraction technique exploits, ideally, a single long (typically few picoseconds) electron bunch in which time-varying structure information is encoded after photo-excitation of diffraction samples being probed by electron diffraction $[1,2]$. The long electron bunch acts as an observable time-window of the dynamics from which different temporal components are separated in space under a transient electric field generated inside the streak camera. Here, we present an optical fiber-based ultrafast lowenergy electron gun developed for time-resolved streak electron diffraction. We find a new robust mean to actively control the electron bunch length by exploiting dispersion effects of the optical fiber used for the photocathode substrate, and therefore, opening the possibility to optimize the time-window for time-resolved streak electron diffraction by tailoring geometrical parameters of the fibre and its coupling to the laser beam. Compared to conventional methods (for example, RF field induced bunch broadening or pulse stretching by using grating pairs) to deliver the picosecond long photoinjection pulses stretched from femtosecond laser pulses, our electron gun concept is relatively simple and combines all these features in a fiber optic based device.

\section{Experimental}

The optical fibre-based photocathode [3] is prepared by direct coating of $30 \mathrm{~nm}$ gold on the polished surface of the metallic fibre ferrule in which the multimode fibre is connecterized by using epoxy glue as shown in Fig. 1(a), (b), (c). The core and cladding diameter of the fibre is selected to $100 \mu \mathrm{m}$ and $125 \mu \mathrm{m}$, respectively, for the efficient coupling between the free space UV pulse to the fibre end. The gold-coated fibre ferrule is mounted to a ferrule

*Corresponding author: dwayne.miller@mpsd.mpg.de 
holder (Fig. 1 (d)) in which three electrostatic Einzel lens plates are stacked with three isolation plates (Fig. 1 (e) and Fig. 2 (a)). The lens aperture diameter and the plate thickness are chosen optimally, based on results from the particle tracing simulator (Fig. 2 (b)), CST particle studio ${ }^{\circledR}[4]$. During the experiment, the number of electrons per bunch is fixed to 200.

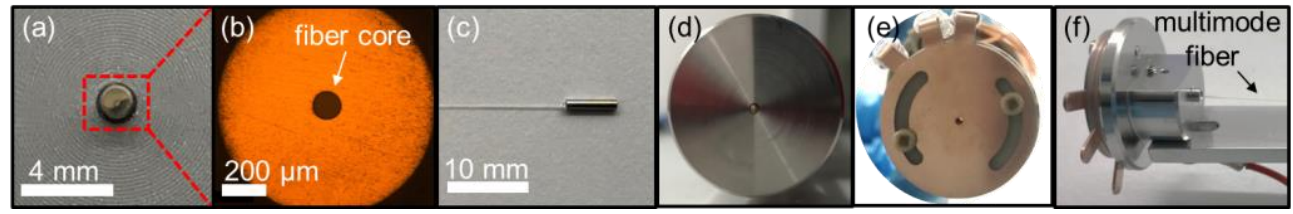

Fig. 1. View of the optical fiber-based electron gun. (a) gold coated fiber ferrule, (b) optical microscope image of the magnified view of the selected region in (a), (c) fiber ferrule connecterized with the fiber, (d) ferrule holder before the assembly of Einzel lens plates, (e) Einzel lens plates, (f) the entire gun assembly.

Fig. 2 (c) and (d) show the variation of the electron beam spot size at the detector screen, depending on the biasing voltage applied to the lens plate at the fixed cathode voltage of $-1.6 \mathrm{kV}$. As shown in Fig. 2 (e) the rms beam spot size at the detector is reduced by more than the factor of two in the case of maximum focusing condition that corresponds to the lens voltage of $-1.22 \mathrm{kV}$, compared to that of $-0.8 \mathrm{kV}$. This beam size reduction gives rise to the increase of the maximum pixel intensity by a factor of 15 . Detailed analysis on the profile of the maximum focused beam reveals that the beam shape fits well to a Gaussian distribution, indicating the minimal spatial aberration of the Einzel lens system.
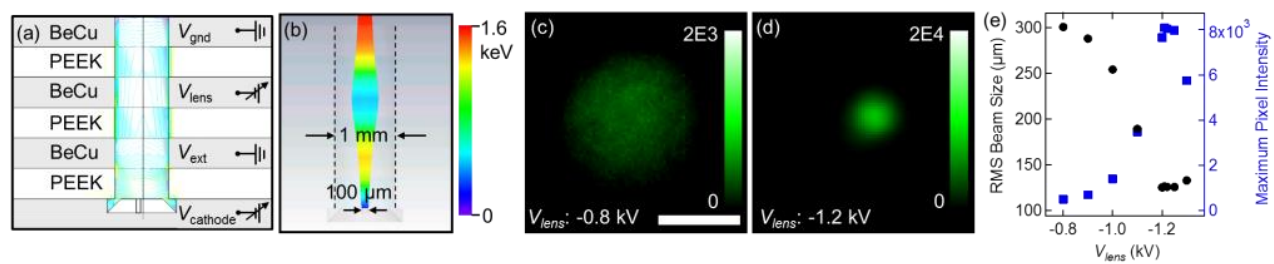

Fig. 2. View of the optical fiber-based electron gun. (a) gold coated fiber ferrule, (b) optical microscope image of the magnified view of the selected region in (a), (c) fiber ferrule connecterized with the fiber, (d) ferrule holder before the assembly of Einzel lens plates, (e) Einzel lens plates, (f) the entire gun assembly.

In order to characterize the bunch length of the electron beam, we used a streak camera generating a transient electric field in the orthogonal direction with respect to the electron beam propagation direction [5]. The streaked electron beam spot image (Fig. 3 (b)) is compared with the unstreaked one (Fig.3 (a)), with a regularization technique, providing typical double-humped temporal profile of the electron bunch as shown in Fig. 3 (c). The FWHM bunch length is evaluated as $14 \pm 2$ ps.

When the laser trigger pulse for the electron emission travels through a waveguide, the temporal pulse width stretches owing to dispersion effects. Given the geometrical parameters, and the coupling condition, the FWHM temporal width of the stretched pulse along the travel length the fiber used in the present work $(1 \mathrm{~m})$ is estimated as $13.69 \mathrm{ps}$. We note that this estimated triggering pulse width is close to the measured electron bunch length, leading us to conclude that space-charge or initial electron kinetic energy spread induced bunch broadening is negligible for the electron bunch generated in the fiber-based cathode upon its propagation.

In order to verify our scenario, we simulated the temporal electron bunch length using the ASTRA code [6] capable of tracking space charge fields by varying the pulse width of the stretched photoinjection pulse at the end of the fiber and the number of electrons per bunch. As shown in Fig. 4 (d), no perceptible temporal broadening is calculated for the 
bunch composed of 200 electrons, indicating that the space charge effect is negligible for the temporal property of the non-dense electron bunch triggered by the stretched pulse in the range of the FWHM pulse width from 2.4 ps to 14.1 ps. In contrast, for bunches with more than 104 electrons, the bunch length starts to broaden upon propagation even in the case of maximally stretched pulses in the simulation (Fig. 4 (e)). As summarized in Fig. 4 $(\mathrm{g})$, this bunch broadening tendency is significant as the number of electrons per bunch becomes larger and when the pulse width of the trigger is shorter.
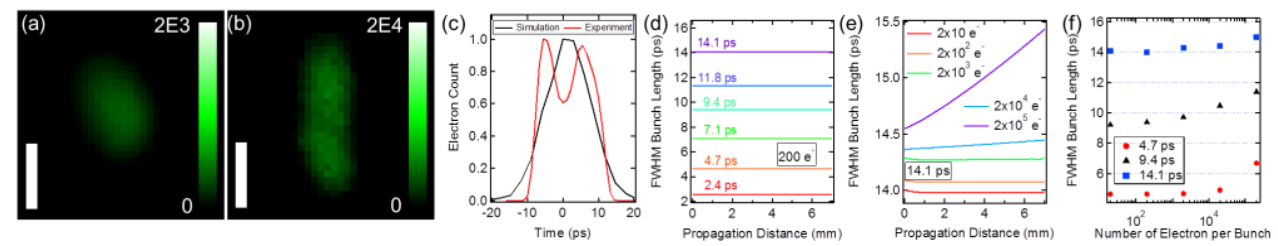

Fig. 3. (a) Temporal characteristics of the electron bunch. For the experiment and simulation, the electron energy was set to $1.6 \mathrm{keV}$. (a) Unstreaked and (b) streaked electron beam image. The scale bar indicates 10 pixels. The intensity profile of these two images are binned horizontally and deconvolved each other with Tikhonov regularization parameter of 2.4 to generate the temporal profile of the electron bunch shown in (c). (d) Calculated FWHM length of the bunch as a function of the trigger pulse width (with 200 electrons), and (e) the number of electrons per bunch (with $14.1 \mathrm{ps}$ trigger pulse) (f) Calculated FWHM length of the bunch measured at the diffraction sample position (5 $\mathrm{mm}$ far from the electron gun).

\section{Summary}

In summary, we have demonstrated an optical fiber-based electron gun integrated with an electrostatic Einzel lens system developed for the purpose of ultrafast streak electron diffraction. Temporal characteristics of the generated electron bunch and simulation results indicate that the temporal length of the non-dense electron bunch triggered by few picosecond laser pulse stretched inside the fiber is minimally affected by space charge effects. The picosecond electron bunch provides the optimal time window to catch entire dynamics of diffraction samples, and can be streaked to decode the information on sub-ps time scale. Therefore, the combination of this relatively long electron pulse duration and steaking technique enables the study of surface-sensitive samples, the ideal target for this low-energy electron probe, with femtosecond resolution in low-energy electron diffraction experiments.

\section{References}

1. M. Eichberger, N. Erasmus, K. Haupt, G. Kassier, A. von Flotow, J. Demsar, and H. Schwoerer, Appl. Phys. Lett. 102, 121106 (2013)

2. P. Musumeci, J. T. Moody, C. M. Scoby, M. S. Gutierrez, M. Westfall, and R. K. Li, J. Appl. Phys. 108, 114513 (2010).

3. A. Casanandruc, R. Bucker, G. Kassier, and R.J.D. Miller, Appl. Phys. Lett. 109, 091105 (2016)

4. See http://www.cst.com

5. G. H. Kassier, K. Haupt, N. Erasmus, E. G. Rohwer, H. M. V. Bergmann, H. Schwoerer, S.M.M. Coelho, and F.D. Auret, Rev. Sci. Instru. 81, 105103 (2010)

6. See http://www.desy.de/mpyflo/ 